\title{
FEDSM2009-78075
}

\section{CFD SOFTWARE APPLICATIONS FOR TRANSCRITICAL FREE SURFACE FLOW}

\author{
Saira F. Pineda \\ Simón Bolívar University \\ Graduate Student \\ Caracas, Venezuela \\ 08-86532@usb.ve
}

\author{
Armando J. Blanco \\ Simón Bolívar University \\ Department of Mechanics \\ Caracas, Venezuela \\ ajblanco@usb.ve
}

\author{
Luis Rojas-Solórzano \\ Simón Bolívar University \\ Department of Energy Conversion \\ Caracas, Venezuela \\ rrojas@usb.ve
}

\begin{abstract}
Flows in rivers, floodplains and coastal zones are very complex due to uneven bottom topography and irregular boundaries of the flow domain. In particular, when the flow shows strong gradients in water depth and velocity it is very difficult to predict, with accuracy, flow characteristics such as water profiles in all points of the domain.
\end{abstract}

Traditional approaches solve shallow-water flow equations, known as Saint-Venant equations, when one or two dimension solutions can be adequate for obtaining most of the important flow characteristics. However, complex situations can require solving NavierStokes equations. In these cases, a two-phase flow problem must be solved and, as water profiles are not known in advance, only a numerical approach can be used to obtain approximate solutions. In addition, flow can be subcritical, supercritical or in a mixed-flow regime. These flow characteristics and complex geometries can make the use of in-house developed software difficult. The arrival of high performance computers and commercial software packages offers new possibilities in the field of numerical hydraulics. However, commercial software packages should be tested on some specific cases; so that these can be used with confidence. In this paper we solve, several cases of free surface flow that consider subcritical, supercritical, critical, oscillatory depth profiles and hydraulic jumps using a commercial package, CFX ${ }^{\mathrm{TM}}$.

Most of these cases were proposed as benchmark solutions by MacDonald et al. (1997) for non-prismatic cross section, non-uniform bed slope and transition between subcritical and supercritical flow. Hydraulic jump cases consist of experimental data of hydraulics jumps obtained by Gharangik \& Chaudhry (1991) for incident flow with Froude numbers of 2.3 and 4.23. In all simulated cases flow was described using a homogeneous model for each phase of the flow.

Turbulence was modeled by using the well-known k- $\varepsilon$ model. In addition, sensitivity to turbulence level in the entrance of flow domain was done to assure independence of results with this variable. Experimental facilities were properly represented in order to assure exact correspondence between boundary conditions of the model and the actual facility.

Results obtained with CFX ${ }^{\mathrm{TM}}$ show excellent agreement with analytical solutions, for subcritical, supercritical, transitional and hydraulic jump cases. Special care with grid selection and entrance boundary condition is crucial to simulate with accuracy these types of flows. In particular, when a proper structured mesh is used, quality results are highly improved. Finally, results show to be insensitive to entrance turbulence conditions.

$\begin{array}{ll}\text { NOMENCLATURE } \\ \mu: & \text { dynamic viscosity of fluid } \\ \rho: & \text { density of fluid } \\ p: & \text { pressure } \\ g: & \text { specific weight } \\ a_{x, y, z}: & \text { acceleration in each direction } \\ V_{x, y, z}: & \text { velocity in each direction } \\ L: & \text { characteristic length in the fluid field } \\ V: & \text { average velocity at cross section } \\ \text { Fr: Froude } & \text { Number }\end{array}$


$T:$ Width of free surface

$Y m$ : average depth

$n$ : Manning Number

d: roughness

$S_{0}$ : bed slope

$y(x)$ : analytical free surface

$U(y)$ : velocity profile

$h$ : depth at inlet

$y$ : depth

$P$ : wet perimeter

\section{INTRODUCTION}

Multiphase flows are common events in nature and in practical applications. In some cases, the phases are separated by a distinct interface which is known as free surface. Flow in waves, river current and rain water above surfaces are some examples of this kind of phenomenon. Also, from a practical point of view it is interesting to study the behavior of flows over channels, drains in roofs, parking zones and waves in ports. Free surface flow can be a destructive phenomenon in nature, as seen by strong rains and floods. These kinds of events can destroy structures and take human lives.

For all these reasons, it is very important to be able to predict free surface flow behavior. Thus, for example, diverse cases to design civil structures and delimit high risk zones can be evaluated. Taken into account the large number of scenarios that need to be considered, numerical modeling is an ideal approach to analyze free surface flows.

Traditional approaches solve shallow-water flow equations, known as Saint-Venant equations, when one or two dimension solutions can be adequate for obtaining most important flow characteristics. However, three dimensional complex situations can require solving Navier-Stokes equations for multiphase flow.

Flow can be subcritical, supercritical or in a mixedflow regime. These flow characteristics and complex geometries can make the use of in-house developed software difficult. The arrival of high performance computers and commercial software packages offers new possibilities in the field of numerical hydraulics. There are important advances in numerical simulation with commercial software: complex geometries can be represented with precision, different turbulence models are available and boundary conditions can be easily imposed. However, the use of full 3D software packages is not so extended when free surface flows must be modeled.

From the author's point of view, the international scientific community is skeptical about the capability of commercial package software to correctly simulate free surface flow properly. As a result, commercial software packages must be carefully tested in specific test cases that include diverse flow regimes and transcritical flows in order to validate the precision of numerical schemes and increase their confidence.

However, one of the most important reasons for the distrust of commercial software is the lack of analytical solutions and reliable experimental data to compare the numerical predictions. This is due to the difficulty of measuring and controlling all the parameters involved in an experiment, like the friction factor of bed slope in channels.

Some analytical solutions to specific subcritical, supercritical and critical flow have been developed for the Saint-Venant equations. These equations are obtained by a process that consists of the integration of Navier-Stokes equations in the orthogonal (and vertical) coordinate of the flow. Therefore, we propose as an alternative way to validate numerical models solving the Navier-Stokes equations doing the comparison with the analytical solutions for specific cases of flow that can be modeled by using the Saint-Venant equations.

In this study, commercial software CFX ${ }^{\mathrm{TM}}$ was used. Several cases proposed by MacDonald et al. (1997) [6] as benchmark solutions for non-prismatic cross section, non-uniform bed slope and transition between subcritical and supercritical flow were modeled. In order to complete the validation process for transcritical flow, hydraulic jumps were modeled. In particular, hydraulic jump cases consist of experimental results data obtained by Gharangik \& Chaudhry (1991) [3] for incident flow with Froude numbers ranging between 2.3 and 4.23.

In all simulated cases, flow was described using a homogeneous model for each phase of the flow. Turbulence was modeled by using the well-known $\mathrm{k}-\varepsilon$ model. In addition, sensitivity to turbulence level in the entrance of flow domain was done to assure independence of results with this variable. Experimental facilities were properly represented in order to assure exact correspondence between boundary conditions of the model and the actual facility, when the simulation of hydraulic jumps was considered.

Since the simulated cases are three dimensional, it was necessary to consider empirical relations between Manning coefficient $n$ (use for modeling $1 \mathrm{D}$ and 2D flows) and the channel wall roughness $d$ [11].

In this paper, the governing equations are first presented, followed by the numerical model scheme. Then, the different considered situations are described. Finally, comparisons with analytical solutions and experimental data show the quality of numerical results obtained from the numerical model. Concluding comments end the discussion. 


\section{MATHEMATICAL MODEL}

In this work, the flow dynamics is modeled by numerically solving the Reynolds Averaged NavierStokes (RANS) equations for the mixture. Flow was considered homogeneous, so, both phases share the same pressure and velocity fields. Turbulence was considered by using the $k-\varepsilon$ model. Sensitivity to turbulence level in the entrance of flow domain was done to assure independence of results with this variable.

To model the air-water segregated flow, the mass conservation of each phase is solved, while the momentum equation (RANS) for each phase are added up to get rid of the interphase momentum transfer term. There is a closure equation for the volume fraction, which states that both phases volume fraction must add up to one at every fluid cell.

The homogeneous multiphase model considers the possibility of air-water mixture at a larger scale than molecular, while still at smaller scale than what is being solved for; i.e., each phase is treated as an interpenetrating continuum, which implies that each phase may be present in every control volume and, the phase volume fraction is equal to the fraction of volume occupied. Therefore, the problem is solved in an Eulerian-Eulerian frame of reference for the two-phases, even though the intrinsic volumetric forces (e.g., gravity) will dictate, through mass conservation, the solely existence, co-existence or non-existence of a single phase in every control volume.

Both phases are considered to be incompressible and isothermal. Therefore, the governing equations are presented, indicating with the sub-index each phase, as follows:

\section{Mass Conservation:

$$
\nabla \bullet\left(r_{\alpha} \rho_{\alpha} U_{\alpha}\right)=0
$$

\section{RANS:}

$\frac{\partial}{\partial t}(\rho U)+\nabla \cdot\left(\left(\rho U \otimes U-\left(\mu+\mu_{T}\right)\left(\nabla U+(\nabla U)^{T}\right)\right)\right)=(B-\nabla p)$

Furthermore, in this equation:

$$
\begin{gathered}
U_{\alpha}=U_{\beta}=U \\
p_{\alpha}=p_{\beta}=p \\
\rho=\sum_{\alpha=1}^{2} r_{\alpha} \rho_{\alpha} \\
\mu=\sum_{\alpha=1}^{N p} r_{\alpha} \mu_{\alpha}
\end{gathered}
$$

And the algebraic restriction for all volume fractions at each control volume:

$$
\sum_{\alpha=1}^{2} r_{\alpha}=1
$$

The equations of the turbulent model are:

Transport of Turbulent Kinetic Energy k :

$$
\nabla \bullet\left(r_{\alpha}\left(\rho_{\alpha} U_{\alpha} k_{\alpha}-\left(\mu+\frac{\mu_{T \alpha}}{\sigma_{k}}\right) \nabla k_{\alpha}\right)\right)=r_{\alpha} S_{k \alpha}
$$

Transport of the Dissipation Rate of Turbulent Kinetic Energy $\varepsilon$ :

$$
\nabla \bullet\left(r_{\alpha}\left(\rho_{\alpha} U_{\alpha} \varepsilon_{\alpha}-\left(\mu+\frac{\mu_{T \alpha}}{\sigma_{\varepsilon}}\right) \nabla \varepsilon_{\alpha}\right)\right)=r_{\alpha} S_{\varepsilon \alpha}
$$

Where the respective source terms are given by:

$$
\begin{aligned}
& S_{k \alpha}=P_{\alpha}+G_{\alpha}-\rho_{\alpha} \varepsilon_{\alpha} \\
& S_{\varepsilon \alpha}=\frac{\varepsilon_{\alpha}}{k_{\alpha}}\left(C_{1 \varepsilon}\left(P_{\alpha}+C_{3 \varepsilon} \max \left(G_{\alpha}, 0\right)\right)-C_{2 \varepsilon} \rho_{\alpha} \varepsilon_{\alpha}\right)
\end{aligned}
$$

The turbulent viscosity is calculated through the Prandtl-Kolmogorov relationship:

$$
\mu_{T \alpha}=C_{\mu} \rho_{\alpha} \frac{k_{\alpha}^{2}}{\varepsilon_{\alpha}}
$$

And the empirical coefficients (taken for free turbulence cases) given by: $\mathrm{C}_{\mu}=0.09 ; \mathrm{C}_{1}=1.44 ; \mathrm{C}_{2}=1.92$; $\mathrm{C}_{3}=0.0$; and $\mathrm{C}_{\mathrm{k}}=0.4187$.

Further details of the mathematical model may be found in the ANSYS-CFX User's manual.

Different flow regimes could appear in free surface flow. Depending on the value of the Froude number, flow behavior could be very different. The Froude number $F_{r}$ is a non dimensional number that represents the relation between the inertial forces and the gravitational forces in a fluid field. It is defined in each point of the flow as [7]:

$$
F r=\frac{V}{\sqrt{g L}}
$$

where $V$ is the local velocity of the flow, $g$ the gravity acceleration and $L$ is the local depth of the flow. Flow could be subcritical $\left(F_{r}<1\right)$, critical $\left(F_{r}=1\right)$ and supercritical $\left(F_{r}<1\right)[14]$.

Typical boundary conditions for Navier-Stokes equations are non-slip velocity condition between flow and solid boundaries, hydrostatic pressure in outlets and mass flow in inlets. 
Depending on the shape of the cross section, channels can be classified as regular cross sections channel and irregular cross sections channels [7]. The regular cross sections are the ones that do not change their shape along the main flow direction. In this paper, the study cases are made with regular cross sections.

For practical purposes in 1D and 2D mathematical models such as the Saint-Venant equations, particular bottom effects are usually taken into account by the definition of a coefficient that allows shear stress at the surface to be included. In particular, the most popular approach consist in representing the bottom effects through the Manning coefficient $n$ which is an empirically determinated value that depends on the relative roughness of the bed slope. Typical values of the Manning coefficient have been determined for a large number of bottom surfaces [11].

However, when Navier-Stokes equations must be numerically solved, the bottom characteristics are defined by the particular relative roughness of the surface. Henderson [5] shows an empirical equation that related the Manning coefficient $n$ to roughness $d$.

$$
n=0.031 d^{1 / 6}
$$

where $d$ is expressed in feet. Others authors such as Strickler [7] propose another empirical equation

$$
n=0.034 d^{1 / 6}
$$

In this work, both expressions and the typical values of Manning coefficient were tested, and finally the last one were chosen because numerical predictions were closer to analytical solutions for the ensemble of cases analyzed.

\section{NUMERICAL MODEL}

All governing equations of Free Surface Flow, with appropriate boundary conditions, were solved in a Cartesian coordinate system using the CFD commercial software ANSYS-CFX ${ }^{\mathrm{TM}}$. Heat transfer was neglected and steady state flow was considered in all cases.

A multi-block unstructured non-uniform grid was used to discretize the entire computational domain. Grids with hexahedral and tetrahedral elements were tested. Numerical results with hexahedral elements were better than those obtained when tetrahedral elements were used in terms of computing time, stability and accuracy [13]; therefore, the former types of elements were selected for this study.

For the grid construction, mesh seeds were located along the axial direction of the model and in the transversal face of the domain, hexahedral elements were then generated.
In order to select the appropriate grid element density for the entire flow condition of interest, several grids were considered. The free surface representation was compared between different grids. A grid was considered adequate once the free surface depth kept a relative difference of less than $3 \%$ when compared to the result obtained by using a refined grid.

The direction perpendicular to the bed slope is that which requires more precision when simulating the free surface and to properly capture the velocity gradient. Therefore, particular efforts in grid refining were made in this direction.

Boundary conditions imposed at each boundary were: (a) Inlet: the average velocity for each phase, (b) Outlet: Static pressure; a hydrostatic distribution for water phase and a pressure reference value for air, (c) Wall: non-slip condition with a corresponding rough wall; this condition is imposed on the bed slope and on both lateral walls, (d) Opening: static pressure reference value; this condition is imposed on the top of the domain. Figure 1 shows boundary conditions in each face of the flow domain.

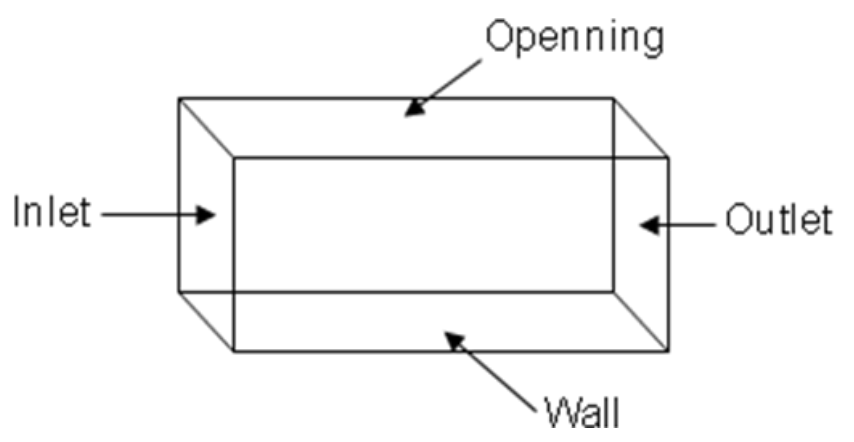

Figure 1. Boundary conditions in the channel's geometry

The bottom relative roughness $d$ was obtained by using empirical Manning Coefficients from White F. M. [11] and the equations (14) and (15) as it was established before.

Spatial resolution was second order of precision. Convergence criteria of $10^{-6}$ for each scaled residual component in velocity and the pressure field was demanded to consider that steady state solution had been reached.

\section{CASES UNDER STUDY}

To validate the commercial software in open channels flow with nonuniform bed slope, analytical solutions from MacDonald et al. [6] and experimental results for hydraulics jumps from Charangik and Chaudhry [3] were compared to numerical predictions. Subcritical flow in rectangular and trapezoidal cross sections channels, transition from subcritical to 
supercritical flow, and from supercritical to subcritical (hydraulic jumps) flows were considered.

Each test has an analytical expression for the bed slope and the free surface. The latter is the expression to be compared later.

Knowing the bed slope and the cross section, the geometry is built.

Case 1: Flow in an open channel of rectangular cross section, in subcritical flow regime.

An open channel with rectangular cross section; $1000 \mathrm{~m}$ long and $10 \mathrm{~m}$ wide was modeled. The Manning coefficient is 0.03 . The flow rate at outflow is $20 \mathrm{~m}^{3} / \mathrm{s}$, the flow regime is subcritical at inflow and outflow. The bed slope is given by $S_{0}(x)[6]$ :

$S_{0}(x)=\left[1-\frac{4}{g y(x)^{3}}\right] y^{\prime}(x)+0,36 \frac{[2 y(x)+10]^{4 / 3}}{[10 y(x)]^{10 / 3}}$

where the analytical solution for the free surface depth and its first derivative are represent by $y(x)$ and $y^{\prime}(x)$ expressed as [6]

$y(x)=\left(\frac{4}{g}\right)^{1 / 3}\left\{1+\frac{1}{2} \exp \left[-16\left(\frac{x}{1000}-\frac{1}{2}\right)^{2}\right]\right\}$

$y^{\prime}(x)=-\left(\frac{4}{g}\right)^{1 / 3} \frac{2}{125}\left(\frac{x}{1000}-\frac{1}{2}\right) \exp \left[-16\left(\frac{x}{1000}-\frac{1}{2}\right)^{2}\right]$

Case 2: Flow in an open channel of rectangular cross section, in subcritical and supercritical flow regime.

An open channel with rectangular cross section; 1000 $\mathrm{m}$ long and $10 \mathrm{~m}$ wide was simulated. The Manning coefficient is 0.02 . The flow rate at outflow is $20 \mathrm{~m}^{3} / \mathrm{s}$, the flow regime is subcritical at inflow and supercritical at outflow. The bed slope is given by $S_{0}(x)[6]$

$S_{0}(x)=\left[1-\frac{4}{g y(x)^{3}}\right] y^{\prime}(x)+0,16 \frac{[2 y(x)+10]^{4 / 3}}{[10 y(x)]^{10 / 3}}$

where the analytical solution for the free surface depth and its first derivative are represent by $y(x)$ and $y^{\prime}(x)$ expressed as [6] $y(x)=\left\{\begin{array}{ll}\left(\frac{4}{g}\right)^{1 / 3}\left\{1-\frac{1}{3} \tanh \left[3\left(\frac{x}{1000}-\frac{1}{2}\right)\right]\right\} & 0 \leq x \leq 500 \\ \left(\frac{4}{g}\right)^{1 / 3}\left\{1-\frac{1}{6} \tanh \left[6\left(\frac{x}{1000}-\frac{1}{2}\right)\right]\right\} & 500<x \leq 1000\end{array}\right\}$

$y^{\prime}(x)=\left\{\begin{array}{ll}-\left(\frac{4}{g}\right)^{1 / 3} \frac{1}{1000} \sec h^{2}\left[3\left(\frac{x}{1000}-\frac{1}{2}\right)\right] & 0 \leq x \leq 500 \\ -\left(\frac{4}{g}\right)^{1 / 3} \frac{1}{1000} \sec h^{2}\left[6\left(\frac{x}{1000}-\frac{1}{2}\right)\right] & 500<x \leq 1000\end{array}\right\}$

Case 3: Flow in an open channel of trapezoidal cross section, in subcritical flow regime.

An open channel with trapezoidal cross section was considered. The free surface width is given by $T=10+4 y$ and the wet perimeter is $\mathrm{P}=10+2 y \sqrt{5}$, the channel is $5000 \mathrm{~m}$ long. The Manning coefficient of the bottom is 0.03 . The flow rate at outflow is $20 \mathrm{~m}^{3} / \mathrm{s}$, the flow regime is subcritical both at inflow and outflow. The bed slope is given by $S_{0}(x)[6]$ :

$S_{0}(x)=\left\{1-\frac{400[10+4 y(x)]}{g[10+2 y(x)]^{3} y(x)^{3}}\right\} y^{\prime}(x)+0,36 \frac{[10+2 y(x) \sqrt{5}]^{1 / 3}}{[10+2 y(x)]^{1 / 3} y(x)^{10 / 3}}$

where the analytical solution for the free surface depth and its first derivative are represent by $y(x)$ and $y^{\prime}(x)$ represented as [6]:

$y(x)=\frac{9}{8}+\frac{1}{4} \sin \left(\frac{\pi x}{500}\right)$

$y^{\prime}(x)=\frac{\pi}{2000} \cos \left(\frac{\pi x}{500}\right)$

Case 4: Hydraulic jumps: transition from supercritical and subcritical flow regime

Once the validation of the $\mathrm{CFX}^{\mathrm{TM}}$ software is completed for these three cases of flow in open channels with changes in bed slope and cross section, the numerical models to validate the commercial software for hydraulic jumps considering different Froude numbers were built. Experimental data from laboratory experiments [3] were used to validate the CFX ${ }^{T M}$ predictions for hydraulic jumps.

The boundary conditions imposed were exactly the same as previously explained, except at inflow. Numerical predictions of the place where the hydraulic jump occurs and the water depths, both upstream and downstream, were very sensitive to the velocity profile at the domain inlet. In consequence, several tests with changes to the velocity profile were made in order to establish better inflow boundary conditions. 
Three different velocity profiles were tested: a constant velocity profile, the parabolic velocity profile (23) and the Couette velocity profile (24).

$$
\begin{aligned}
& u(y)=\frac{3 \bar{V}}{h^{2}}\left(h y-\frac{y^{2}}{2}\right) \\
& u(y)=\frac{6 \bar{V}}{h^{2}}\left(h y-y^{2}\right)
\end{aligned}
$$

The effect of the velocity profile on the free surface numerical predictions can be observed in figure 2 for $F_{r}=2.3$.

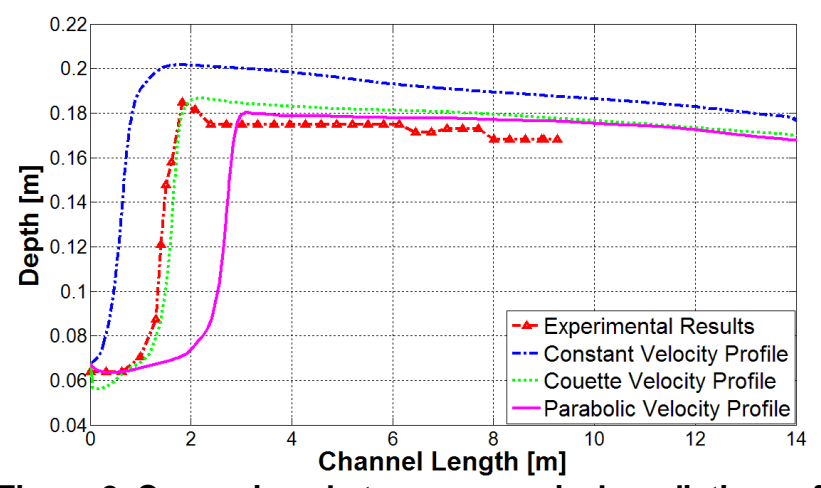

Figure 2. Comparison between numerical predictions of free surface position for different velocity profile at the inlet $\left(F_{r}=2.30\right)$

It is observed how the numerical results are very sensitive to the inflow boundary condition at inflow. Because none of these results were considered satisfactory, the actual laboratory facility was reproduced. [3].

The experimental set consists of an open channel and a tank as shown in figure 3. The domain has a gate, which is located between the tank and the open channel. The open channel is $14 \mathrm{~m}$ long, $0.915 \mathrm{~m}$ high and 0.46 $\mathrm{m}$ wide. For the simulation the Manning coefficient is 0.008 with an equivalent roughness of $0.00017 \mathrm{~m}$. The tank is $0.46 \mathrm{~m}$ wide, $1.5 \mathrm{~m}$ high and $0.40 \mathrm{~m}$ long. Changes in the gate position resulted in different types of flows, and in consequence, flows with different Froude number were obtained [3].

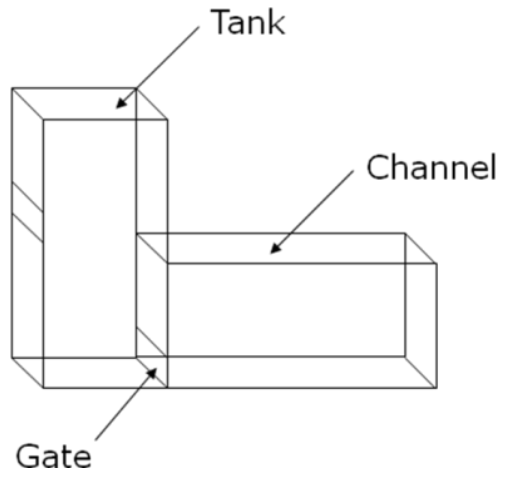

Figure 3. Geometry used to simulate hydraulic jump

The particular cases of hydraulic jumps characterized by Froude numbers of, 2.30 and 4.23 were considered. Table 2 shows the particular values for each case.

Table 1. Validation cases for hydraulic jumps

\begin{tabular}{|c|c|c|c|c|}
\hline Case & $\begin{array}{c}\text { Froude } \\
\text { number }\end{array}$ & $\begin{array}{c}\text { Opening } \\
\text { Gate High } \\
(\mathbf{m})\end{array}$ & $\begin{array}{c}\text { Velocity } \\
(\mathbf{m} / \mathbf{s})\end{array}$ & $\begin{array}{c}\text { Flow } \\
\text { Rate } \\
\left(\mathbf{m}^{3} / \mathbf{s}\right)\end{array}$ \\
\hline $4 \mathrm{a}$ & 2.3 & 0.0064 & 1.826 & 0.05376 \\
4b & 4.23 & 0.043 & 2.737 & 0.05414 \\
\hline
\end{tabular}

Next section present the comparison between numerical predictions and analytical solutions (when it is possible) and experimental results.

\section{RESULTS} slope

Results for open channel flow with irregular bed

In this work, the comparison to validate the $\mathrm{CFX}^{\mathrm{TM}}$ software for subcritical and supercritical flow regimes with different and variable bed slope and different cross section was made using the analytical expression of free surface for each case previously described.

Figures 4 and 5 show the comparison between analytical solutions given by (a) equation (17) for subcritical pure flow and (b) equation (20) and regime transition between subcritical and supercritical flow. The numerical results show an excellent definition of free surface in comparison to the analytical solution. Both curves are in the same position, which means that the software is able to accurately represent the free surface. 


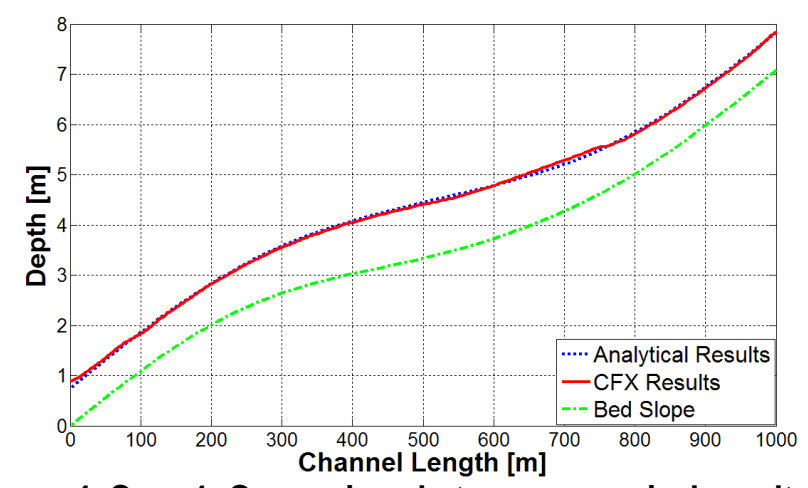

Figure 4. Case 1: Comparison between numerical results and the analytical solution for rectangular cross section in subcritical flow regime

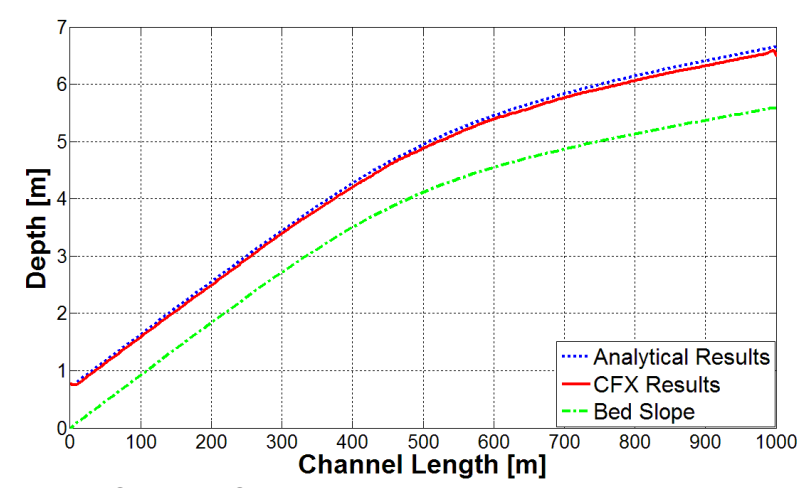

Figure 5. Case 2: Comparison between numerical results and the analytical solution for rectangular cross section in transition between subcritical and supercritical regime

Numerical results show only a maximum deviation of $0.1 \%$ (case 1 ) and $1.75 \%$ (case 2). So, it is concluded that the numerical solution is able to simulate free surface flow in open channels with rectangular cross section, with different bed slope in subcritical (case 1) and transcritical regimes (case 2).

In order to extend the research to channels with a cross section different from the rectangular one, an open channel with a trapezoidal cross section and bed elevation with high variations was considered in case 3 .

As it is shown in figure 6 , numerical results obtained from $\mathrm{CFX}^{\mathrm{TM}}$ show the same trend as the analytical solution (21). In fact, the free surface is able to follow the continuous changes in bottom shape. Free surface coincides both in phase and amplitude with the analytical expression.

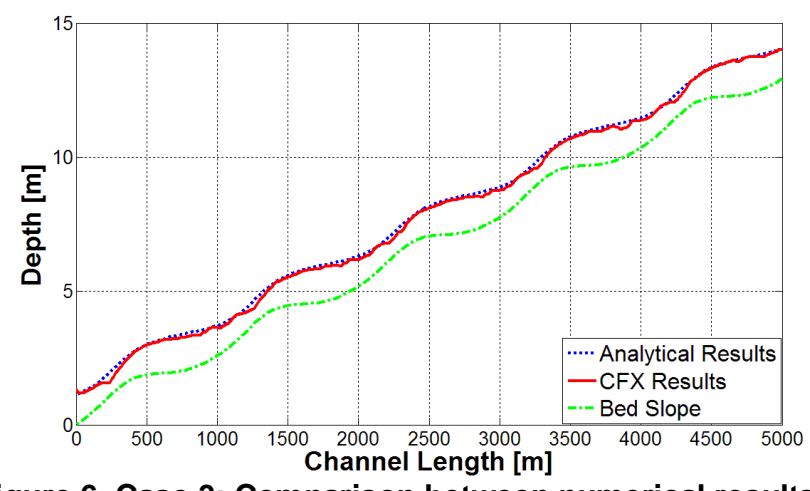

Figure 6. Case 3: Comparison between numerical results and the analytical solution for trapezoidal cross section and highly variable bottom elevation in subcritical regime

The numerical results show a maximum difference of $2 \%$ with respect to the analytical solution. This means that the numerical model is able to simulate free surface flow in highly variable bottom conditions and non-trivial cross section. For practical purposes, the difference between numerical results and the analytical solution is neglected from a practical engineering point of view.

\section{Results for hydraulic jumps cases}

Case 4a: $F_{r}=2.30$

Figure 7 shows the comparison between numerical results and experimental data for this hydraulic jump with $F_{r}=2.30$. The numerical results agree with the experimental data, both upstream and capturing the position where the hydraulic jump appears.

Some differences are obtained downstream. Despite of these differences the results are highly satisfactory; these results are almost the same order of magnitude as the error in experimental measurements $(1 \mathrm{~cm})$. In addition, a hydraulic jump is not static, so, experimental measurements of the free surface positions are estimated values. The difference between numerical results and the experimental ones is around $5 \%$ at the location of the point where the depth reaches its maximum value. Therefore, numerical results can be considered very satisfactory. 


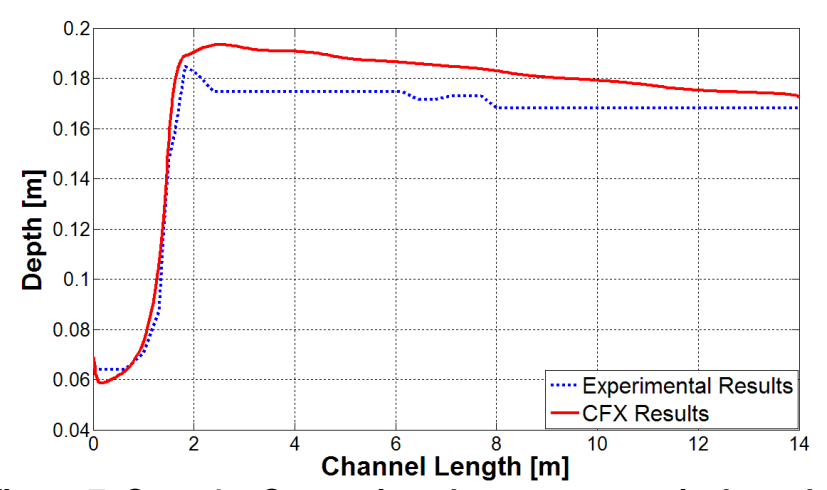

Figure 7. Case 4a: Comparison between numerical results and experimental for hydraulic jump with $F_{r}=2,30$

Case 4b: $F_{r}=4.23$

Figure 8 shows the comparison between numerical results and experimental data for this hydraulic jump with $F_{r}=4.23$. Numerical results show the correct location of the maximum point of the hydraulic jump; it also shows a very acceptable representation of the free surface flow at the point where the hydraulic jump takes place.

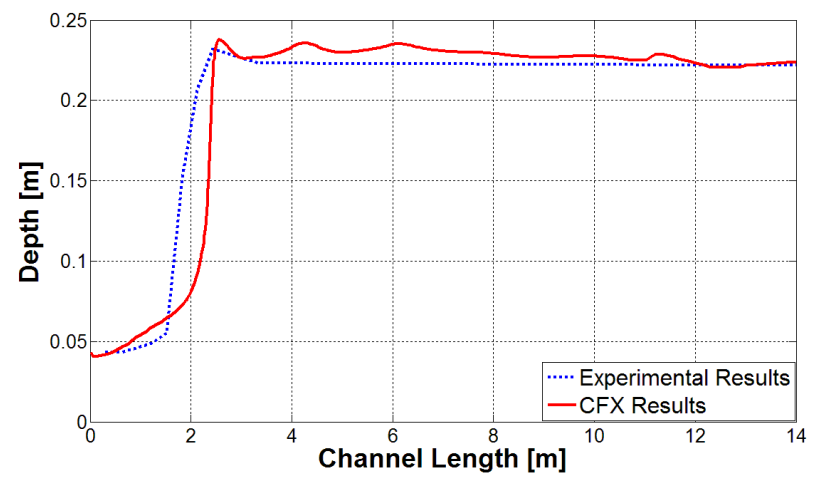

Figure 8. Case 4b: Comparison between numerical results and experimental for hydraulic jump with $F_{r}=4.23$

It is important to mention that as the Froude Number increases, the hydraulic jump is more turbulent. This presents more oscillations in the free surface, and, in consequence, a steady state is very difficult to be reached.

The difference between the numerical results and the experimental ones is $3 \%$ at the location of maximum point of the hydraulic jump. This difference is negligible for reasons explained above.

For higher Froude numbers, the flow is not steady state and for this reason, numerical results were considered as being not representative of the actual situation. However, despite the fact that as the Froude Number increases the numerical results have more difference with respect to the experimental data, in most cases, this difference is small and from an engineering point of view, is negligible.
Finally, in order to analyze the effect of the inlet turbulence intensity on the numerical results a parametric study with this variable was done. Numerical results proved to be not sensitive to variation in the inlet turbulence level.

\section{CONCLUSIONS}

The results obtained in the validation of the $\mathrm{CFX}^{\mathrm{TM}}$ software are very satisfactory in the simulation of free surface flow in steady state situations. The commercial software $\mathrm{CFX}^{\mathrm{TM}}$ is able to simulate open channels with changes in bed slope and different cross sections, both rectangular and trapezoidal. $\mathrm{CFX}^{\mathrm{TM}}$ can be used for simulating flow in subcritical regime and transitions between subcritical and supercritical regimes. The difference between the numerical results and the analytical solutions are very small and negligible from a practical point of view for engineering applications.

The selection of the correct mesh is crucial to obtain good results. To simulate free surface flow the mesh that gives better results is the mesh made by hexahedral elements.

The results obtained in the validation of the $C F X^{T M}$ software are acceptable in the simulation of hydraulic jumps. Correct definition of boundary conditions is very important to obtain accurate results when hydraulic jumps are modeled. CFX ${ }^{\mathrm{TM}}$ is able to determine the exact location where the hydraulic jump takes place and to simulate the position of the point where the water depth reaches the maximum value for flows with low and moderate Froude number.

As the Froude Number increases, it is more difficult to obtain a good agreement between numerical results and experimental data, although, numerical results are acceptable from an engineering point of view. In particular, numerical results show high sensitivity to variations in the inflow boundary condition.

To finalize, the commercial software CFX is able to simulate free surface flow for transcritical flows.

\section{ACKNOWLEDGMENTS}

The author wishes to acknowledge to the FONACIT project number G-2005000507 for supporting this research.

\section{REFERENCES}

1. Bellos, C., Sakkas, J. 1987, 1-D Dam Break FloodWave Propagation on Dry Bed, Journal of Hydraulic Engineering.

2. Blanco, A. J. 2003, Modelos Reológicos para Aluviones Torrenciales, Reporte Interno Proyecto Milenio, Instituto de Mecánica de los Fluidos, Facultad de Ingeniería, Universidad Central de Venezuela. 
3. Charangik, A. M., Chaudhry, M. H. 1991, Numerical Simulation of Hydraulic Jump, Journal of Hydraulic Engineering.

4. Coussot, P. 1997, Mudflow Rheology and Dynamics, A. A. Balkema, Rotterdam.

5. Henderson, F. H. 1966, Open Channel Flow, Macmillan.

6. MacDonald, I., Baines, M. J., Nichols, N. K., Samuels, P. G. 1997, Analytic Benchmark Solutions for Open Channel Flows, Journal of Hydraulic Engineering.

7. Méndez, M. V. 2001, Elementos de Hidráulica de Canales, UCAB, Primera Edición, Caracas.

8. Potter, M. C., Wiggert, D. C. 1998, Mecánica de Fluidos, Prentice Hall, Segunda Edición.
9. Rickenmann, D., Koch, T. 1997, Comparison of Debris Flow Modelling Approaches.

10. Shames, I. H. 1995, Mecánica de Fluidos Mc Graw Hill, Tercera Edición.

11. White, F. M. 2004, Mecánica de Fluidos Mc Graw Hill, Quinta Edición.

12. Hernández F., 2006, BSc in Mechanical Engineering Dissertation Project. Universidad Simón Bolívar Venezuela.

13. Pineda S., 2007, BSc in Mechanical Engineering Dissertation Project. Universidad Simón Bolívar Venezuela.

14. Munson, B., Young, D., Okiishit, T., 2002, Fundamentals of Fluid Mechanics, John Willey \& Sons, USA. 